\title{
3.12 OPTICAL PULSAR IN NGC 4254
}

\author{
P. A. FELDMAN \\ Queen's University, Ontario, Canada
}

Dr. Feldman described the optical variability which has been seen over the years in a region near the nucleus of NGC 4254 (M99) and he suggested that it might be a pulsar.

The following summary has been prepared by the Editors.

Lampland noted in 1921 that NGC 4254 showed signs of variability near its nucleus; these were secular changes in form and brightness. Walker made an investigation of Lampland's plate material for the period 1916 to 1948 and added his own observations in 1966-1967. He confirmed the existence of a variable object about $200 \mathrm{pc}$ from the centre of NGC 4254. It had the characteristics of a blue stellar object with an absolute photographic magnitude of $-14^{\mathrm{m}}$. Walker suggested that it might be a low luminosity quasar.

The present suggestion is that it may be a pulsar with about $10^{7}$ times the luminosity of the Crab Nebula pulsar. If it is assumed that its magnetic field is about one tenth of Crab pulsar and that its efficiency in converting a dipole magnetic field into optical emission is 10 times more than for the Crab pulsar, then the pulsar in NGC 4254 would be easily detectable optically. However its radio flux would be $10^{-2}$ flux units and its X-ray emission would also be too weak to detect. 